\title{
Variations in the local structure of nano-sized anatase $\mathrm{TiO}_{2}$
}

\author{
Helen Y. Playford
}

\section{Published version information}

Citation: HY Playford. "Variations in the local structure of nano-sized anatase $\mathrm{TiO}_{2} . "$ Journal of Solid State Chemistry, vol. 288 (2020): 121414.

DOI: $\underline{10.1016 / \text { i.j.jsc. } 2020.121414}$

(C)2020. This manuscript version is made available under the CC-BY-NC-ND 4.0 Licence.

This version is made available in accordance with publisher policies. Please cite only the published version using the reference above. This is the citation assigned by the publisher at the time of issuing the AAM. Please check the publisher's website for any updates. 


\section{Variations in the Local Structure of Nano-sized Anatase $\mathrm{TiO}_{2}$}

Helen Y. Playford

ISIS Facility, Rutherford Appleton Laboratory, Didcot, UK, OX11 0QX

\section{Abstract}

The structures of three polymorphs of $\mathrm{TiO}_{2}$ (anatase, rutile \& brookite) are examined using neutron diffraction and total scattering. While the short-range structures of (bulk) rutile and brookite show excellent agreement with their average crystal structures, the structure of the anatase samples changes with reduction of particle size. Reverse Monte Carlo (RMC) analysis reveals that a fraction of oxygen atoms in the anatase nanoparticles are displaced away from their ideal positions. The displacement results in a change to the octahedral geometry which is compared with a theoretical structural modification that results in a reduced band gap.

Keywords: structure, disorder, titanium dioxide, neutron diffraction, pair distribution function

\section{Introduction}

Titanium dioxide, $\mathrm{TiO}_{2}$, is one of the most industrially important simple oxides, and has numerous applications as a bright white pigment, an absorber of ultraviolet (UV) light and, notably, a UV-active photocatalyst [1]. There are three commonly occurring polymorphs of $\mathrm{TiO}_{2}$ : anatase, rutile and brookite (Figure 1). Their structures, which are tetragonal (anatase and rutile) or orthorhombic (brookite) consist of differently arranged networks of corner- and/or edge-sharing [ $\left.\mathrm{TiO}_{6}\right]$ octahedra [2]. Of these three polymorphs, rutile is the most stable in bulk form, however there is reportedly a "crossover" size of between 11 and $30 \mathrm{~nm}$ at which surface energy considerations render anatase more stable than rutile [3-5]. As a result of the ease of its preparation in nanosized form, anatase is the most commonly employed for photocatalysis [4], although some reports have found brookite, or a mixture of anatase and brookite, to have a higher intrinsic activity $[4,6]$.

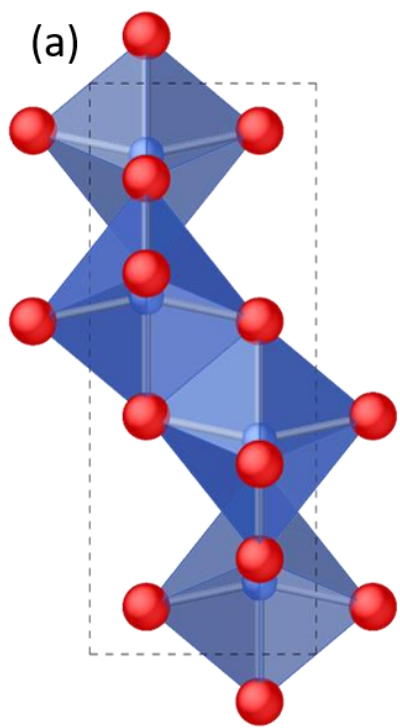

(b)

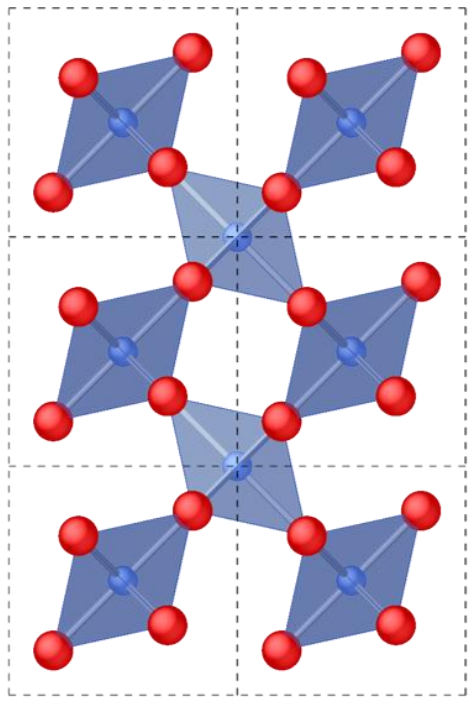

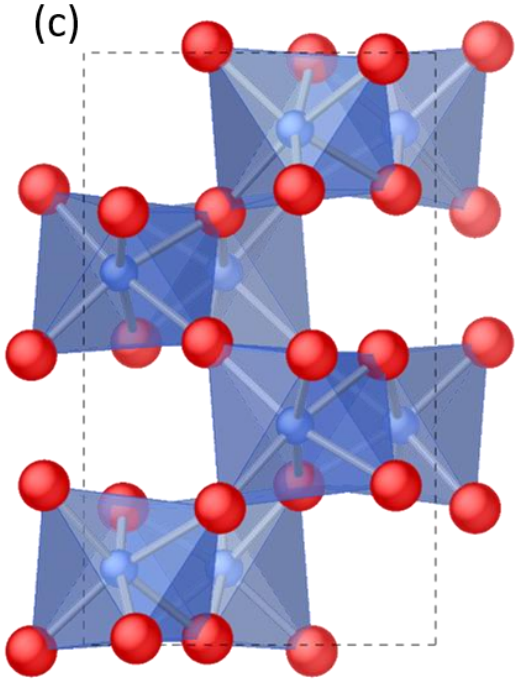

Figure 1: Polyhedral representations of the crystal structures of (a) anatase, (b) rutile and (c) brookite $\mathrm{TiO}_{2}$. Blue spheres/polyhedra are $\mathrm{Ti}$, red spheres are $\mathrm{O}$ and the unit cell edges are shown as dashed black lines.

There are numerous methods for the preparation of nanosized anatase, including hydrothermal synthesis, solgel methods and emulsion techniques [1]. The hydrolysis of titanium alkoxides is a common "soft chemical" 
route to nanosized anatase, however the particle size and purity of the product is influenced by many factors including the choice of alkoxide, amount of water added, $\mathrm{pH}$, aging time and temperature, and annealing conditions [7].

The existence of nanosized anatase in a bulk sample is usually confirmed using X-ray powder diffraction, though the extraction of structural information is hindered because of particle-size-broadened Bragg peaks and the common co-existence of other polymorphs, particularly brookite [8]. Since the atomic structure of the nanomaterials is of great importance to their properties, numerous studies have used local structural analysis techniques such as transmission electron microscopy (TEM), X-ray absorption spectroscopy and Raman spectroscopy.

Raman spectra measured from anatase $\mathrm{TiO}_{2}$ nanocrystals are broadly similar to that of the bulk material, but generally exhibit line broadening and frequency shifts that can be correlated with particle size [9]. Phonon confinement, non-stoichiometry and pressure effects all contribute to these observations, although the relative importance of each contribution is likely to depend on the preparation route [10-12].

A pre-edge feature in the XANES spectra of nanosized $\mathrm{TiO}_{2}$ is usually attributed to the presence of 5-coordinate $\mathrm{Ti}$ sites, and its intensity has been observed to increase with decreasing particle size in proportion to the changing surface area to volume ratio $[13,14]$. Analysis of EXAFS data has shown increased lattice distortion and shorter Ti-O bonds in smaller particles $[15,16]$ and the short bonds were attributed to Ti-OH correlations on the surface [16]. More recently, a combination of multiple techniques, including X-ray diffraction, XANES, Raman spectroscopy and electron microscopy was used by Luca [17] to establish that hydrothermally ripened, sol-gel derived anatase nanoparticles have a core-shell structure which consists of a bulk-like interior and a surface layer with a significant amount of disorder and under-coordinated Ti. It was further suggested that the depth of this layer is sufficient that under ambient conditions, atmospheric water molecules are prevented from accessing and completing the co-ordination of all of the unsaturated $\mathrm{Ti}$.

Total scattering is a powder-diffraction based technique in which Bragg and diffuse scattering are measured and analysed together. The measured data are carefully normalised and corrected for the effects of nonsample scattering to produce the total scattering function, $F(Q)$, which can be Fourier transformed to produce the pair distribution function (PDF), $G(r)$ [18-20]. The PDF can be thought of as a weighted histogram of the interatomic distances within a material, and analysing it is a powerful method by which to probe local structure. The real-space resolution of total scattering data is dependent upon the range of momentum transfer, $\boldsymbol{Q}$, over which the reciprocal space data are measured. Since the magnitude of $\boldsymbol{Q}$ is inversely proportional to wavelength, $\lambda$, high energy radiation is required to maximise this value. Therefore the best total scattering data are obtained from high-energy synchrotron X-rays and spallation-source neutrons. The increased availability of these types of sources in recent years has led to an increase in the number of applications of total scattering, and the technique, which had its origins in the study of liquids and glasses, has now been used to provide insight into the local structures of many disordered crystalline materials [21-24]. Typically, modelling total scattering data involves either a "small-box" or a "large-box" approach. Small-box modelling is a method analogous to Rietveld refinement where the parameters of a crystal structure are refined against the PDF rather than the Bragg diffraction pattern, whereas large-box modelling involves the calculation of scattering functions from a large atomic configuration (in the case of a crystalline material, usually a supercell of the crystal structure) followed by many millions of random atomic moves until an acceptable fit between simulated and measured scattering data is obtained. Large-box modelling can also be referred to as a reverse Monte Carlo (RMC) approach. Both techniques have distinct advantages and disadvantages, and are particularly powerful when used in combination. 
Rather few applications of total scattering to $\mathrm{TiO}_{2}$ have been reported, which is surprising given the importance of local atomic structure and structural disorder to understanding its properties. The majority of existing studies focus on nanosized or amorphous materials. So-called amorphous titania appears to be a material with considerable structural variety, with one RMC study [25] finding it to resemble a defective form of brookite and another [26] determining that it consisted of core-shell nanoparticles with recognizable anatase cores and highly distorted shells. Several studies have considered the evolution of structure in $\mathrm{TiO}_{2}$ nanoparticles during synthesis, for example, in situ X-ray PDFs collected during hydrothermal synthesis [27] revealed a precursor consisting of clusters of $\mathrm{TiO}_{5} / \mathrm{TiO}_{6}$ units arranged similarly to the anatase structure. The combination of XANES/EXAFS with X-ray PDFs used to study the effect of thermal treatment on microemulsion $\mathrm{TiO}_{2}$ [28] also found a precursor structure related to anatase, but containing $\mathrm{TiO}_{5}$ units. Differences in the stacking of $\mathrm{TiO}_{5} / \mathrm{TiO}_{6}$ units were found responsible for the observed differences in local order between samples. The morphology of titania nanoparticles has also been correlated to industrially relevant properties such as lithium diffusion rates and catalytic behaviour, through the use of Debye function analysis [29], differential evolution refinement [30] and small-box modelling of the PDF [31].

In this work we report the application of neutron total scattering and RMC modelling to crystalline anatase samples of different particle size, in order to obtain insight into structural variations that are likely to be highly relevant when determining possible applications of such materials. The use of neutrons for this study is of particular importance because the scattering lengths of $\mathrm{Ti}$ and $\mathrm{O}$ (-3.438 and $5.803 \mathrm{fm}$, respectively) allow excellent "visibility" of both elements as well as excellent contrast between them.

\section{Experimental}

Samples of bulk crystalline and "nanopowder" anatase ( $<25 \mathrm{~nm}$ particle size) $\mathrm{TiO}_{2}$ were obtained from SigmaAldrich and used without further purification. Bulk rutile $\mathrm{TiO}_{2}$ was obtained by heat treatment of bulk anatase at $900{ }^{\circ} \mathrm{C}$ for 12 hours. Brookite $\mathrm{TiO}_{2}$ was synthesised by hydrothermal treatment of $1.0 \mathrm{~mL}$ titanium bis(ammonium lactate)dihydroxide solution ( $50 \mathrm{wt} \%$ in $\mathrm{H}_{2} \mathrm{O}$, Sigma-Aldrich) with $8.0 \mathrm{~mL}$ of $6 \mathrm{M}$ urea solution in sealed Teflon-lined vessels at $160^{\circ} \mathrm{C}$ for 24 hours [32]. Anatase nanoparticles were synthesised by two different sol-gel methods. The first sample, referred to herein as "N1", was prepared by the addition of $10 \mathrm{~mL}$ of titanium isopropoxide ( $97 \%$, Sigma-Aldrich) to a mixture of $60 \mathrm{~mL}$ isopropanol and $3 \mathrm{~mL} \mathrm{D}_{2} \mathrm{O}$, which resulted in the production of a thick white suspension. The suspension was stirred for one hour at room temperature and subsequently the solid product was collected by suction filtration and calcined at $400{ }^{\circ} \mathrm{C}$ for 24 hours. Sample "N2" was produced following the method of Shi et al. [33] wherein a solution of $5 \mathrm{~mL}$ titanium nbutoxide (97\%, Sigma-Aldrich) in $45 \mathrm{~mL}$ ethanol was added dropwise to a rapidly-stirred mixture of $50 \mathrm{~mL} \mathrm{D}_{2} \mathrm{O}$ and $50 \mathrm{~mL}$ ethanol. The resulting suspension was stirred for 12 hours, the solid product was collected by suction filtration and then re-suspended in a further $50 \mathrm{~mL}$ of ethanol. After filtration the solid product was dried at $100{ }^{\circ} \mathrm{C}$ for 10 hours and then calcined at $400{ }^{\circ} \mathrm{C}$ for 2 hours.

The phase purity of all samples was assessed using powder X-ray diffraction on a Rigaku MiniFlex benchtop diffractometer operating with $\mathrm{Cu} \mathrm{K} \alpha$ radiation.

Neutron total scattering data were collected on the Polaris diffractometer at the ISIS neutron and muon source. Samples were loaded into cylindrical vanadium cans of 6 or $8 \mathrm{~mm}$ diameter and placed into the instrument. Data were collected for a total of 8-10 hours per sample to ensure they were of good statistical quality, and additional datasets from an empty can and the empty diffractometer were collected for data reduction purposes. Diffraction patterns suitable for Rietveld refinement were obtained using the program Mantid [34], and the total scattering data were more rigorously normalised and corrected for non-sample scattering using the program GudrunN [20]. Pair distribution functions (PDFs) were obtained via Fourier 
transformation of the corrected scattering data using the program StoG, distributed as part of the RMCProfile package [21]. Rietveld refinements were carried out using the programs GSAS [35] and EXPgui [36], small-box "real-space Rietveld" modelling of extracted PDFs using PDFgui [37], and reverse Monte Carlo (RMC) refinements using RMCProfile [21].

\section{Results \& Discussion}

\subsection{Rietveld refinement}

A summary of Rietveld refinement results for all 6 samples can be seen in Table 1. For every sample, data from all 5 of Polaris' detector banks were used. For each bank a shifted Chebyschev polynomial with 7 parameters was used to fit the background, and GSAS profile function TOF 3 was used to describe the peak shape. The refined structural parameters were lattice parameters, atomic positions (where the atoms did not lie on special positions) and anisotropic (where allowed) thermal displacement parameters. In all cases excellent fits were obtained using single phase models. No evidence of contamination of the brookite sample with anatase, nor of N1 and N2 with brookite was seen. The reduction in particle size in the three nano-sized samples is seen clearly in broadened Bragg peaks. An estimation of particle size of the anatase samples using the Scherrer equation produced values of $280 \mathrm{~nm}$ (bulk), $34 \mathrm{~nm}$ (commercial nanopowder), $18 \mathrm{~nm}$ (N1) and $13 \mathrm{~nm}$ (N2) (see supplementary information for more details on this calculation). The occupancies of the $\mathrm{Ti}$ and $\mathrm{O}$ sites in the anatase samples were refined, but neither deviated meaningfully from unity. This is in agreement with the work of Grey \& Wilson [38] wherein Ti occupancies close to 1 were found in 9-11 nm crystallites. It is clear, therefore, that non-stoichiometry does not have a large part to play in the average structure of these samples.

Table 1: Results of Rietveld refinement of neutron diffraction data for six samples of $\mathrm{TiO}_{2}$.

\begin{tabular}{|c|c|c|c|c|c|c|c|c|}
\hline \multirow{2}{*}{\multicolumn{2}{|c|}{ Sample }} & \multirow[t]{2}{*}{ Bulk rutile } & \multirow{2}{*}{\multicolumn{2}{|c|}{ Brookite }} & \multicolumn{4}{|c|}{ Anatase } \\
\hline & & & & & Bulk & Commercial nanopowder & Synthetic N1 & Synthetic N2 \\
\hline \multicolumn{2}{|c|}{ Space group } & $\mathrm{P}_{2} / \mathrm{mnm}$ & Pbca & & $I 4_{1} / a m d$ & $I 4_{1} /$ amd & $I 4_{1} /$ amd & $I 4_{1} /$ amd \\
\hline \multicolumn{2}{|c|}{ wRp (\%) } & 3.04 & 3.73 & & 2.93 & 2.27 & 2.75 & 3.42 \\
\hline \multicolumn{2}{|c|}{$\chi^{2}$} & 2.564 & 5.343 & & 4.006 & 3.782 & 2.714 & 2.797 \\
\hline \multicolumn{2}{|c|}{$a(\AA ̊)$} & $4.59093(3)$ & $9.1716(5)$ & & $3.78306(3)$ & $3.78356(7)$ & $3.7885(2)$ & $3.7953(3)$ \\
\hline \multicolumn{2}{|c|}{$b(\AA ̊)$} & $=a$ & $5.4469(3)$ & & $=a$ & $=a$ & $=a$ & $=a$ \\
\hline \multicolumn{2}{|c|}{$c(\AA)$} & $2.95706(2)$ & $5.1355(3)$ & & $9.50912(9)$ & $9.4955(2)$ & $9.4808(5)$ & $9.4772(9)$ \\
\hline \multicolumn{2}{|c|}{$V\left(\AA^{3}\right)$} & $62.325(1)$ & $256.55(4)$ & & $136.090(3)$ & $135.931(7)$ & $136.08(2)$ & $136.51(4)$ \\
\hline \multirow[t]{8}{*}{$\mathrm{Ti}$} & $x$ & 0 & $0.1303(2)$ & & 0 & 0 & 0 & 0 \\
\hline & $\mathrm{y}$ & 0 & $0.1028(3)$ & & 0.25 & 0.25 & 0.25 & 0.25 \\
\hline & $\mathrm{z}$ & 0 & $0.8657(3)$ & & 0.375 & 0.375 & 0.375 & 0.375 \\
\hline & Occ. & 1.0 & 1.0 & & 1.0 & 1.0 & 1.0 & 1.0 \\
\hline & $U_{11}$ & $0.00647(9)$ & $0.0028(2)$ & & $0.00461(9)$ & $0.0051(1)$ & $0.0034(2)$ & $0.0029(4)$ \\
\hline & $U_{22}$ & $=U_{11}$ & $=U_{11}$ & & $=U_{11}$ & $=U_{11}$ & $=U_{11}$ & $=U_{11}$ \\
\hline & $U_{33}$ & $0.0039(1)$ & $=U_{11}$ & & $0.00456(13)$ & $0.0054(2)$ & $0.0085(3)$ & $0.0144(5)$ \\
\hline & $U_{12}$ & $-0.0001(1)$ & 0 & & 0 & 0 & 0 & 0 \\
\hline \multirow[t]{3}{*}{0} & $x$ & $0.30487(2)$ & $0.0097(1)^{a}$ & $0.23015(9)$ & 0 & 0 & 0 & 0 \\
\hline & y & $=x$ & $0.1493(2)$ & $0.1122(2)$ & 0.25 & 0.25 & 0.25 & 0.25 \\
\hline & z & 0 & $0.1814(2)$ & $0.5369(2)$ & $0.16698(2)$ & $0.16676(2)$ & $0.16592(4)$ & $0.16500(6)$ \\
\hline
\end{tabular}




\begin{tabular}{rlllllll} 
Occ. & 1.0 & 1.0 & 1.0 & 1.0 & 1.0 & 1.0 & 1.0 \\
$U_{11}$ & $0.00501(3)$ & $0.0052(1)$ & $0.0026(1)$ & $0.00300(8)$ & $0.00280(9)$ & $0.0007(2)$ & $0.0071(21)$ \\
$U_{22}$ & $=U_{11}$ & $=U_{11}$ & $=U_{11}$ & $0.0107(1)$ & $0.0116(2)$ & $0.0126(2)$ & $0.0145(3)$ \\
$U_{33}$ & $0.00366(5)$ & $=U_{11}$ & $=U_{11}$ & $0.00484(6)$ & $0.00534(6)$ & $0.0068(1)$ & $0.0091(2)$ \\
$U_{12}$ & $-0.00223(6)$ & 0 & 0 & 0 & 0 & 0 & 0 \\
Size $(\mathrm{nm})$ & - & - & & $280(61)$ & $34(5)$ & $18(5)$ & $13(3)$ \\
\hline
\end{tabular}

${ }^{\text {a }}$ As there are two $\mathrm{O}$ sites in the brookite structure, this column has been split in two to show two sets of parameters. In all samples $\mathrm{U}_{13}=\mathrm{U}_{23}=0$.

The length of the anatase $c$-axis decreases, and that of the $a$-axis increases with decreasing particle size (Figure 2(a)), which is in agreement with Zhu et al. [39] who observed a similar trend down to particle sizes of approximately $4 \mathrm{~nm}$, but not with Luca [17] who reported an increase in the $c$-axis in particles smaller than $10 \mathrm{~nm}$. As a result of the opposing changes in the two dimensions, the cell volume changes very little in the samples reported in this work, which is in agreement with a large body of literature (as summarised by Luca [17]) that sees a volume increase with decreasing particle size only below $10 \mathrm{~nm}$.

There are two distinct Ti-O bond lengths in the anatase crystal structure, and both sets tend to increase in length as the particle size decreases (Figure 2(b)). The lengthening of the "short" set of bonds is understandable given that they lie close to parallel to the $a b$ plane, but the concurrent lengthening of the "long" set is somewhat paradoxical as they lie along the shortening $c$-axis.
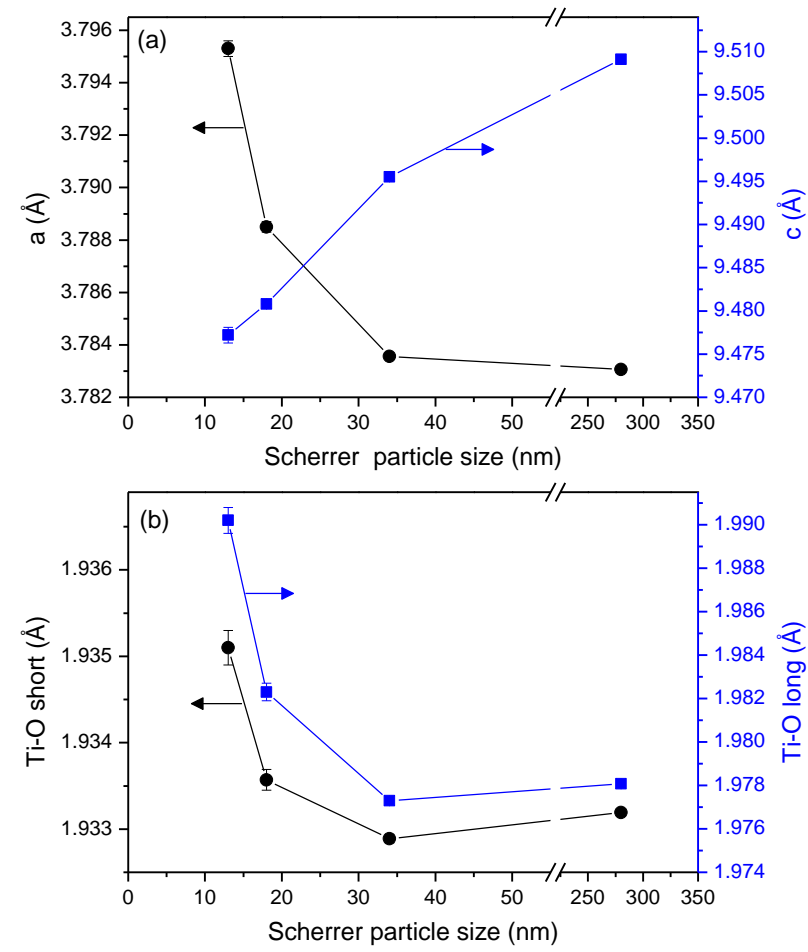

Figure 2: (a) Variation of anatase $\mathrm{TiO}_{2}$ lattice parameters as a function of Scherrer particle size, as determined from Rietveld refinement. If not visible, error bars are contained within the size of the symbols. (b) Variation in the long and short $\mathrm{Ti}-\mathrm{O}$ bond lengths as a function of Scherrer particle size, as determined from Rietveld refinement. If not visible, the error bars are contained within the symbols. In both cases, the circular markers correspond to the left axis, and the square markers to the right axis.

Examination of the thermal ellipsoids obtained from Rietveld refinement reveals that the Ti ellipsoid becomes increasingly elongated in the smaller particles, and the $\mathrm{O}$ ellipsoids become flatter and more disc-like (Figure 3). These highly anisotropic thermal ellipsoids may be indicative of significant levels of static disorder, such as local displacements of Ti atoms or distortions of the octahedral sites. 


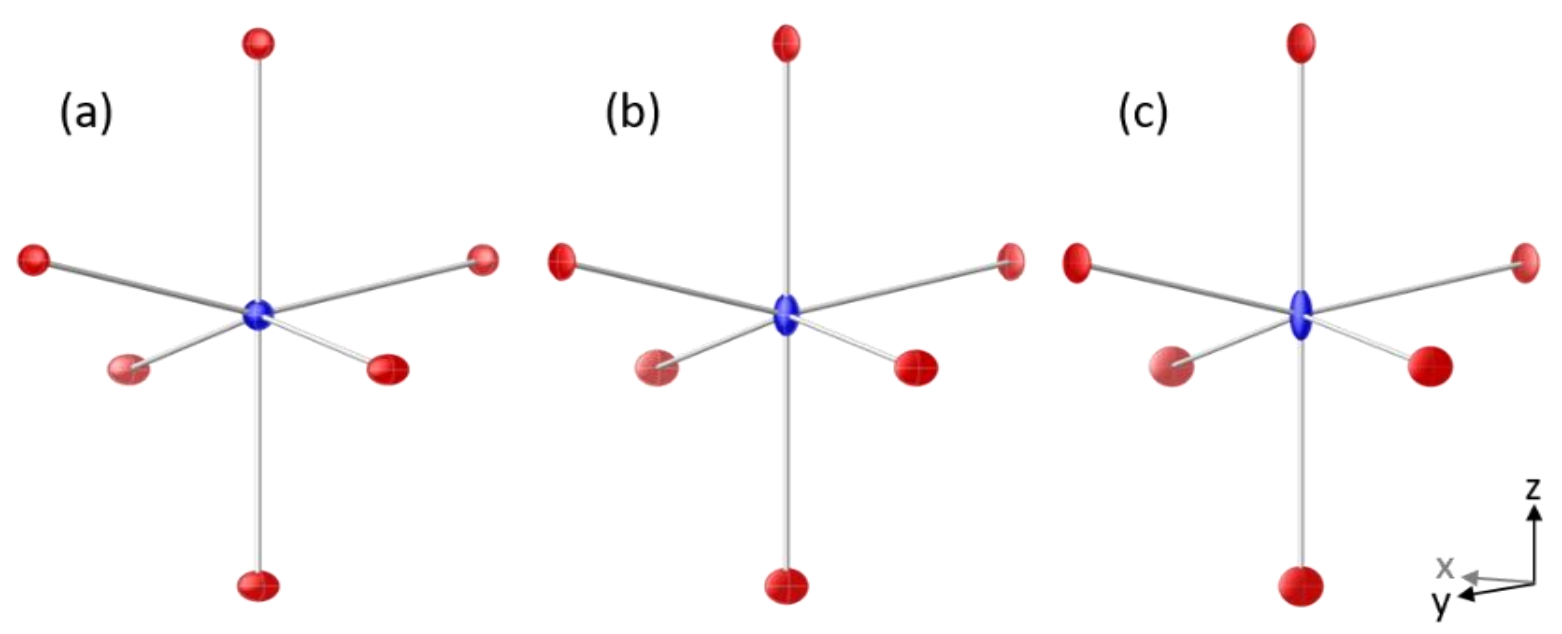

Figure 3: Visualisation of thermal ellipsoids obtained from Rietveld refinement of nanosized anatase samples and plotted at $50 \%$ probability for (a) commercial nanopowder, (b) sample N1 and (c) sample N2. The blue ellipsoids represent the Ti atoms, and the red the $\mathrm{O}$ atoms.

\subsection{Real-space Rietveld}

To determine whether or not the local structure of the $\mathrm{TiO}_{2}$ samples agrees with their average structures, the structures obtained from Rietveld refinement were used as starting models for real-space refinements using PDFgui. In all refinements the $r$-range was $0.02-40 \AA$, and instrument resolution was accounted for using dampening and broadening terms that were refined against data from a standard silicon sample and subsequently fixed. The effect of correlated motion was incorporated by sharpening all PDF peaks below a certain r-cutoff (in this case, $3.38 \AA$ ). . For further details about these parameters, see the PDFgui documentation [37]. In general, the lattice parameters obtained from PDFgui fits differed from those from Rietveld refinement by only a fraction of $1 \%$, and the atomic positional parameters were in similarly good agreement (see Table S1). There were greater discrepancies in the refined thermal parameters, with the PDF refinements usually producing larger ellipsoids. ${ }^{1}$ For both the bulk rutile and brookite samples the final fits were extremely good (Figure S3), and there was no evidence for any discrepancies between average and local structure. For the anatase samples an additional parameter was added to the refinement to provide an estimate of particle size by applying a simple spherical shape function to the calculated PDFs. The fit to the bulk anatase data was excellent (Figure 4), although a clearly erroneous value of $28 \mathrm{~nm}$ was obtained for the particle diameter. This likely arises from the difficulty of separating the effect of particle size from instrumental dampening, as well as the limited $r$-range used for the refinement.

\footnotetext{
${ }^{1}$ The effect of thermal motion on a PDF is to broaden the features, and it is very difficult to separate this broadening from the instrumental contribution which arises from the use of a finite maximum $Q$ in the Fourier transform. Furthermore, there is likely to be some additional correlation between this broadening and the dampening of the PDF that is caused by reciprocal space resolution and, importantly for the nanomaterials, particle size. Therefore it is not possible to interpret these values in great detail.
} 

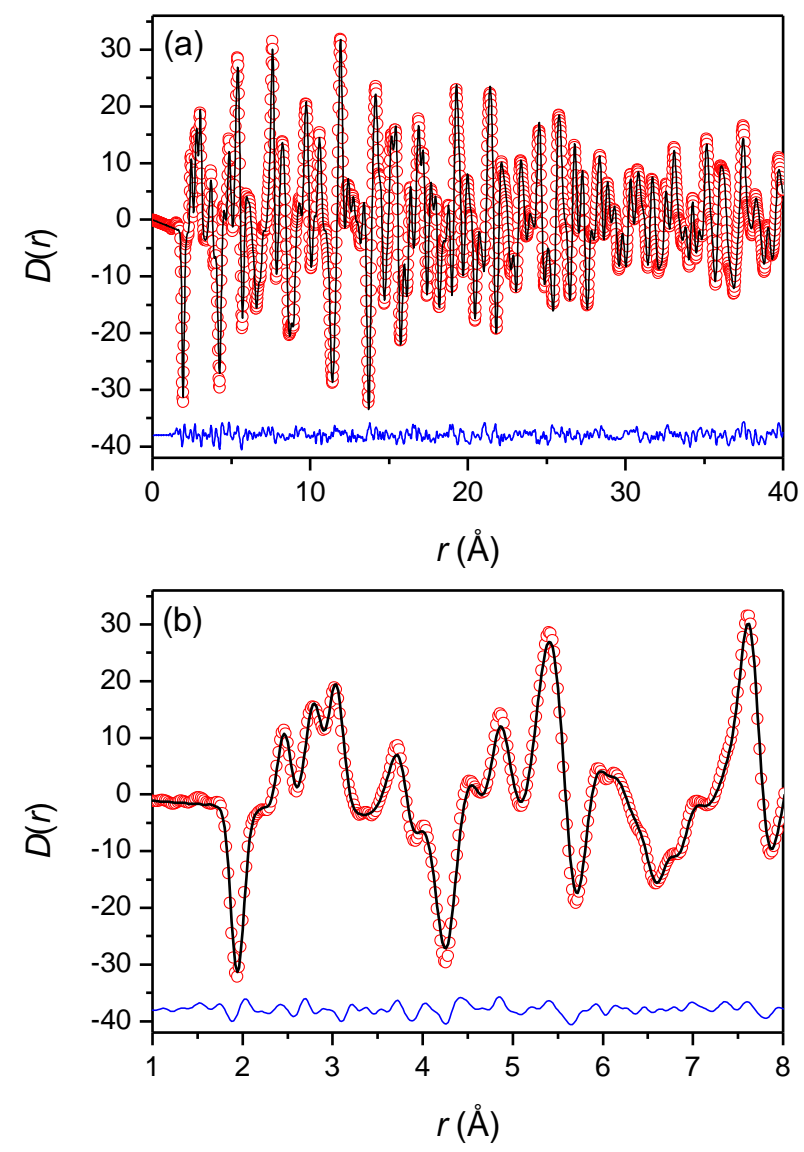

Figure 4: Results of PDFgui "real-space Rietveld" refinement against the neutron pair distribution function from bulk anatase $\mathrm{TiO}_{2}$ with (a) showing the full fitting range and (b) a close-up of the region $1 \AA \leq r \leq 8 \AA$. Red circles are the measured data, the black line is the calculated PDF and the blue line the difference. For this refinement, $\mathrm{Rw}=8.2 \%$ which cannot be directly compared to the residuals from a Rietveld refinement but is nevertheless indicative of an excellent fit.

For the three nanosized anatase samples, the overall quality of the PDF refinements is good (see Supplementary Information, Figure S4), and the refined particle diameters are 18, 12 and $9 \mathrm{~nm}$, respectively. These are reasonably consistent with the values from Scherrer analysis of the Bragg peak width, and are useful as a lower limit on the particle size. It can be seen in Figure 5 that there are some discrepancies between experimental and calculated PDFs in the low- $r$ region that increase in severity with decreasing particle size. The discrepancies are most obvious between $2.25-3.25 \AA$ in the region of the nearest-neighbour O-O correlations, of which there are several due to the distorted geometry of the $\left[\mathrm{TiO}_{6}\right]$ octahedra. It is interesting to note that if there were simply more dynamic disorder in the smaller particles, as is implied by the large thermal ellipsoids on the $\mathrm{O}$ atoms in Figure 3, then you would expect to see only a broadening of the features in the PDF. Instead what is seen are a change in relative intensity of these features, and no obvious change in width, which implies that there may be some distortion in the local structure of the smaller particles that is not captured by the crystal structure. 


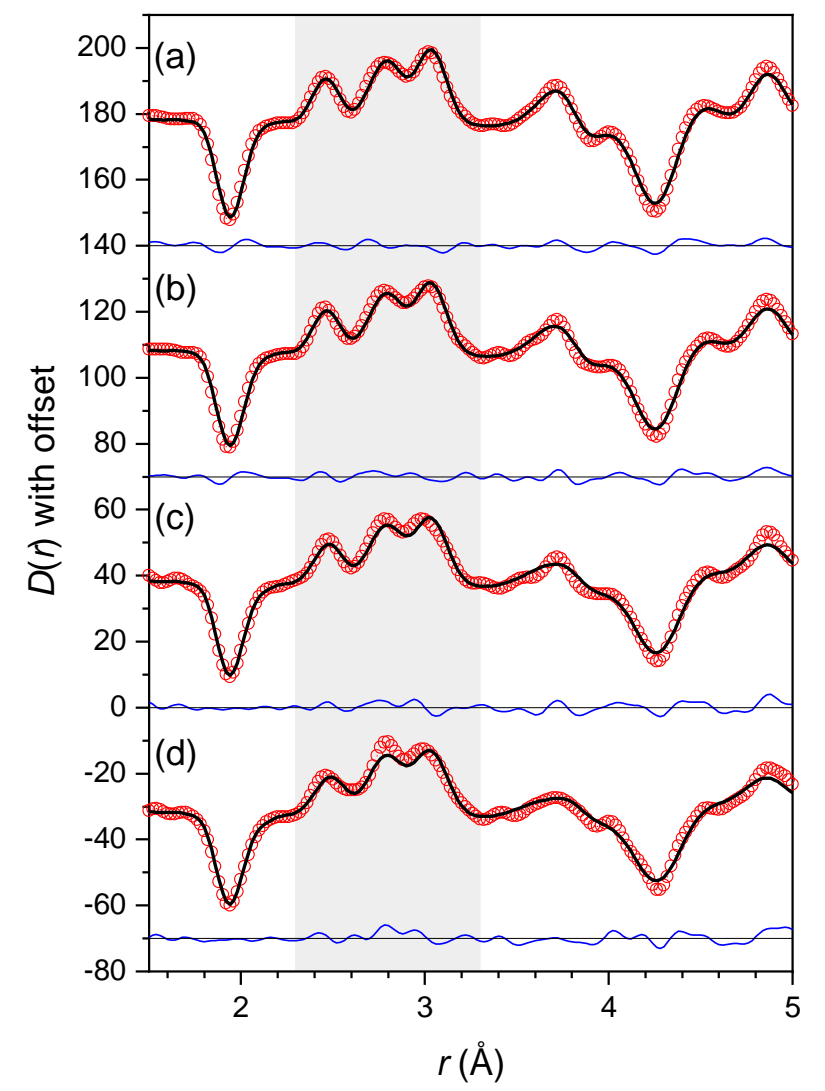

Figure 5: Comparison of the low-r region of the measured and fitted PDFs for (a) bulk anatase, (b) commercial nanopowder, (c) sample N1 and (d) sample N2. Red circles are the measured data, the black lines the calculated PDFs and the blue lines the differences. The grey shaded box highlights the region of the O-O nearest neighbour correlations where the discrepancies in peak intensity are most visible. The $R_{w}$ values for the refinements in the range $1-5 \AA$ are 9.4, 10.2, 12.6 and $13.9 \%$ for (a) - (d) respectively.

\subsection{Reverse Monte Carlo Refinements}

To gain insight into the local disorder evidenced by discrepancies in the PDF fitting and the anisotropic thermal displacement parameters obtained from Rietveld refinement, RMC refinements were carried out. Each refinement used the Rietveld-obtained structure as a starting model, scaled up to $18 \times 18 \times 7$ supercells. The refined atomic configurations were obtained through fitting to three datasets: the Bragg pattern, the PDF and the total scattering function, $F(Q)$. The only additional constraint used was a distance window to maintain the Ti-O connectivity by requiring all Ti-O pairs to fall within the bounds of the first PDF peak. The refinements were run for 4000 minutes ( 3 days), and each was repeated 100 times to build up a large ensemble of configurations from which to calculate statistical quantities such as partial pairdistribution functions. Example PDF and Bragg fits from the bulk anatase and N2 samples are shown in Figure 6, and the full set of fits can be found in Figures S5 and S6. 

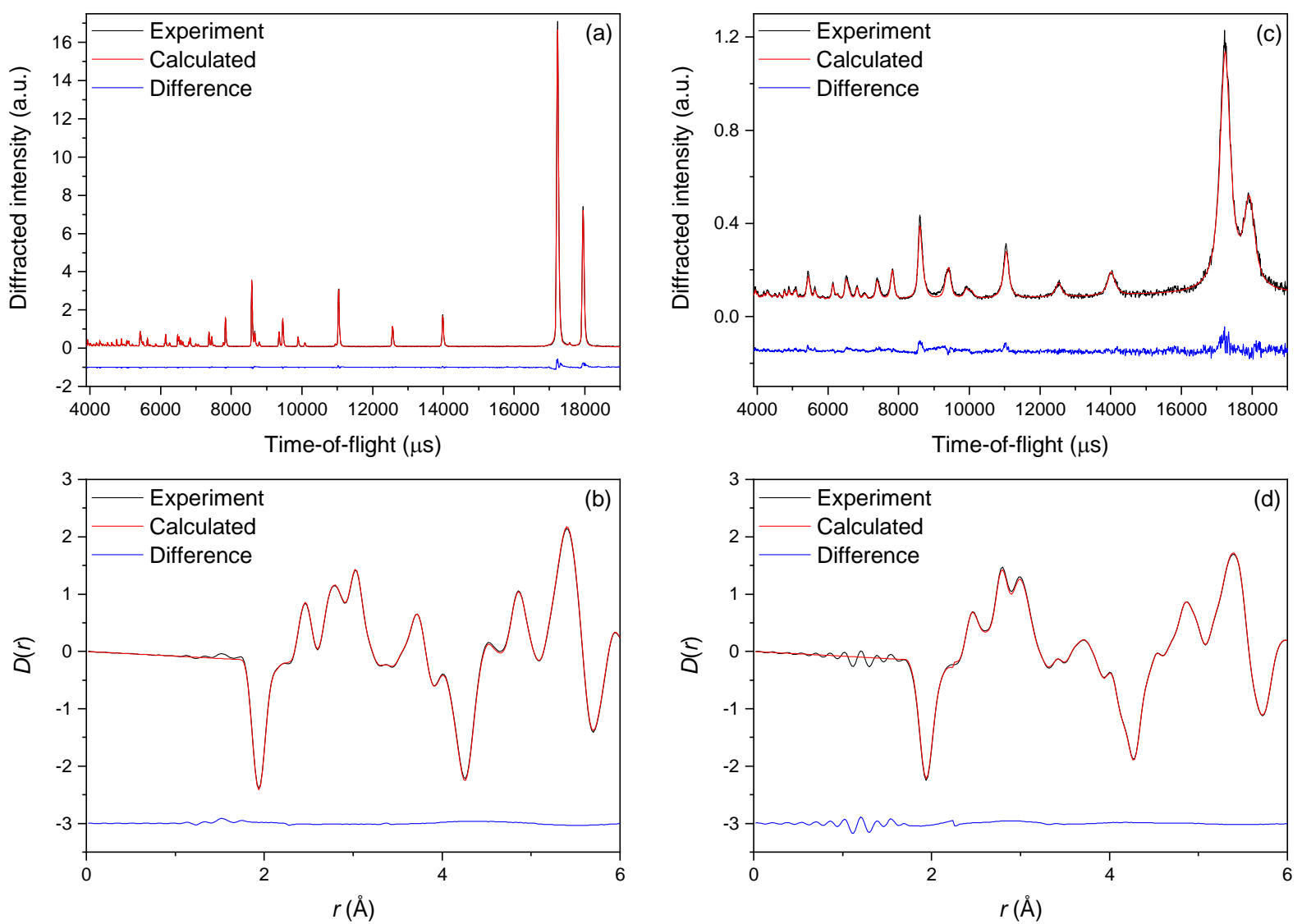

Figure 6: Example RMC fits to the Bragg diffraction pattern and the PDF from (a)-(b) bulk anatase and (c)-(d) sample N2.

Partial pair distribution functions calculated from the RMC refined configurations are shown in Figure 7 and Figure 8. For the $\mathrm{Ti}-\mathrm{O}$ and Ti-Ti pairs, these functions are largely unchanged by reduction in particle size, other than a slight broadening in all but the nearest-neighbour Ti-O peak. Interestingly there is no noticeable shift in the position of the Ti-O nearest-neighbour peak, suggesting that the average bond length is not affected by the changing particle size, although the distribution of "short" and "long" bonds may be different. In the O-O functions, as well as a general broadening indicative of increasing disorder, there is a noticeable shift in the third peak ( $3.0 \AA$ A ) for samples N1 and N2 and an increase in the intensity of the second peak (2.6 $\AA$ ). The partial PDFs indicate that the observed disorder in the smaller nanoparticles is primarily in the oxygen sublattice. 

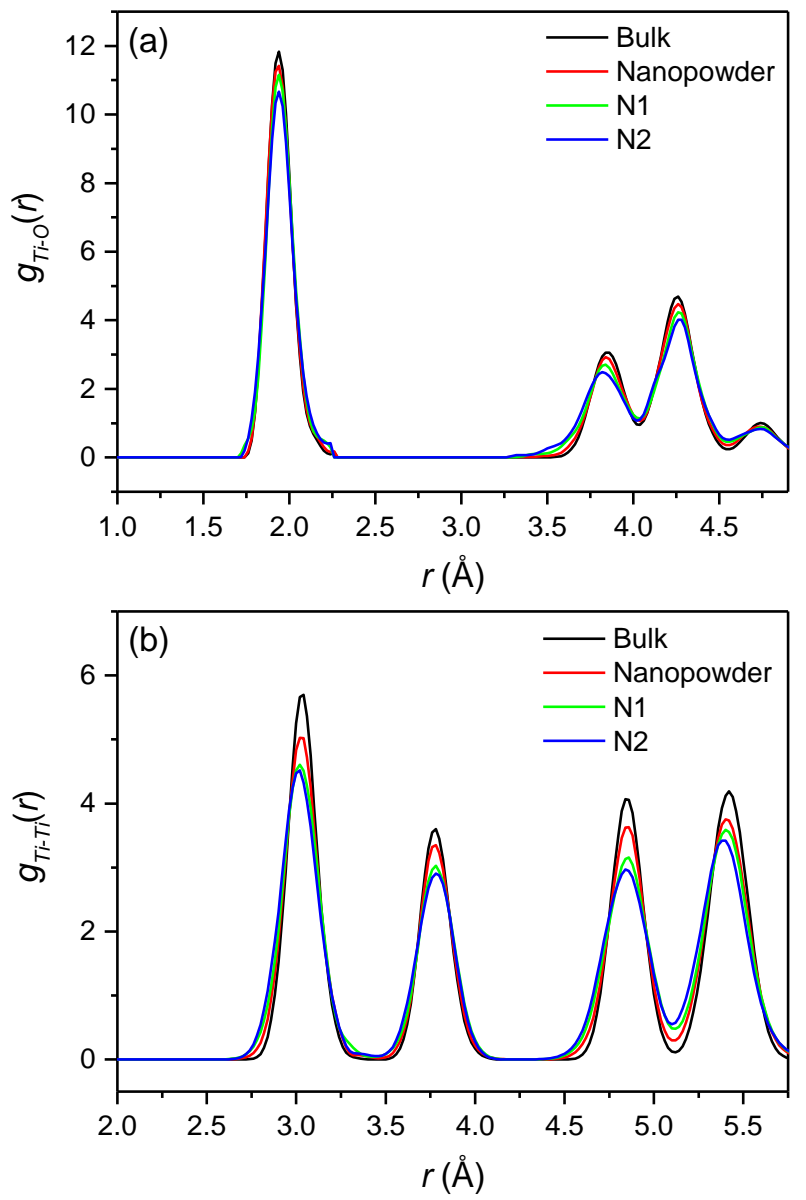

Figure 7: Partial pair distribution functions from the Ti-O (a) and Ti-Ti (b) pairs for the 4 anatase samples, each calculated from an ensemble of $100 \mathrm{RMC}$ refined configurations.

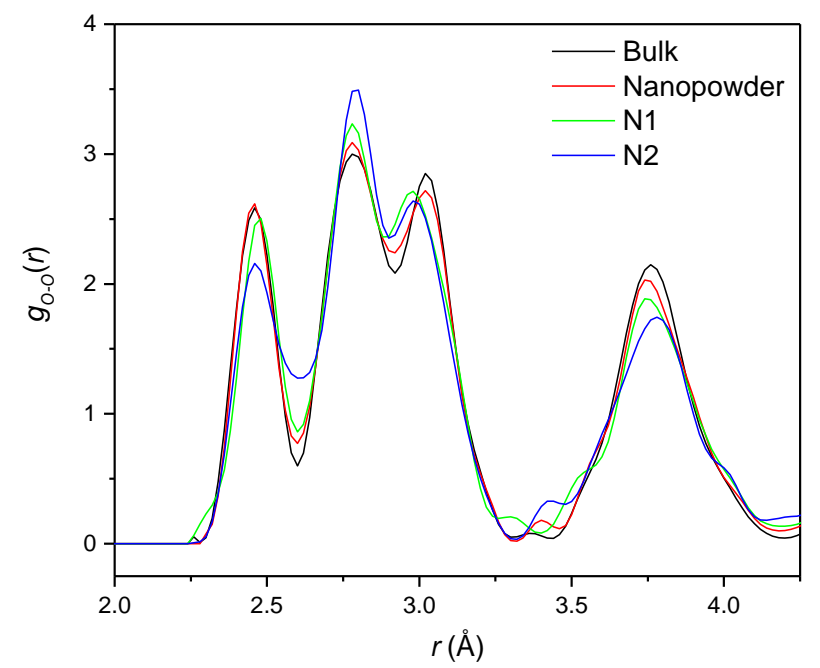

Figure 8: Partial pair distribution functions for the O-O pairs for the 4 anatase samples, each calculated from an ensemble of $100 \mathrm{RMC}$ refined configurations.

Calculated O-Ti-O bond angle distributions and representations of the octahedral geometry are shown in Figure 9. The octahedral environment in anatase is rather distorted with the four "equatorial" oxygen atoms displaced alternately above and below the plane of the $\mathrm{Ti}$ atoms, and this is reflected in the additional peaks in the bond angle distribution. While it can be seen that, for all samples, the positions of the features match the averages from Rietveld refinement, the distributions show a broadening and shift in intensity between $90^{\circ}$ and $100^{\circ}$ that accompanies the reduction in particle size. Figure $\mathrm{S} 7$ shows the bond angle distributions from bulk anatase and sample N2 compared with those from the rutile and 
brookite polymorphs. The octahedra in rutile are more regular with strong maxima at $90^{\circ}$ and $180^{\circ}$, while in brookite the lower symmetry structure leads to greater overlap between the "peaks" in the distribution. There is a strong similarity between the distributions from sample N2 and brookite, suggesting that the smaller nanoparticles have a different octahedral geometry than that in bulk anatase.
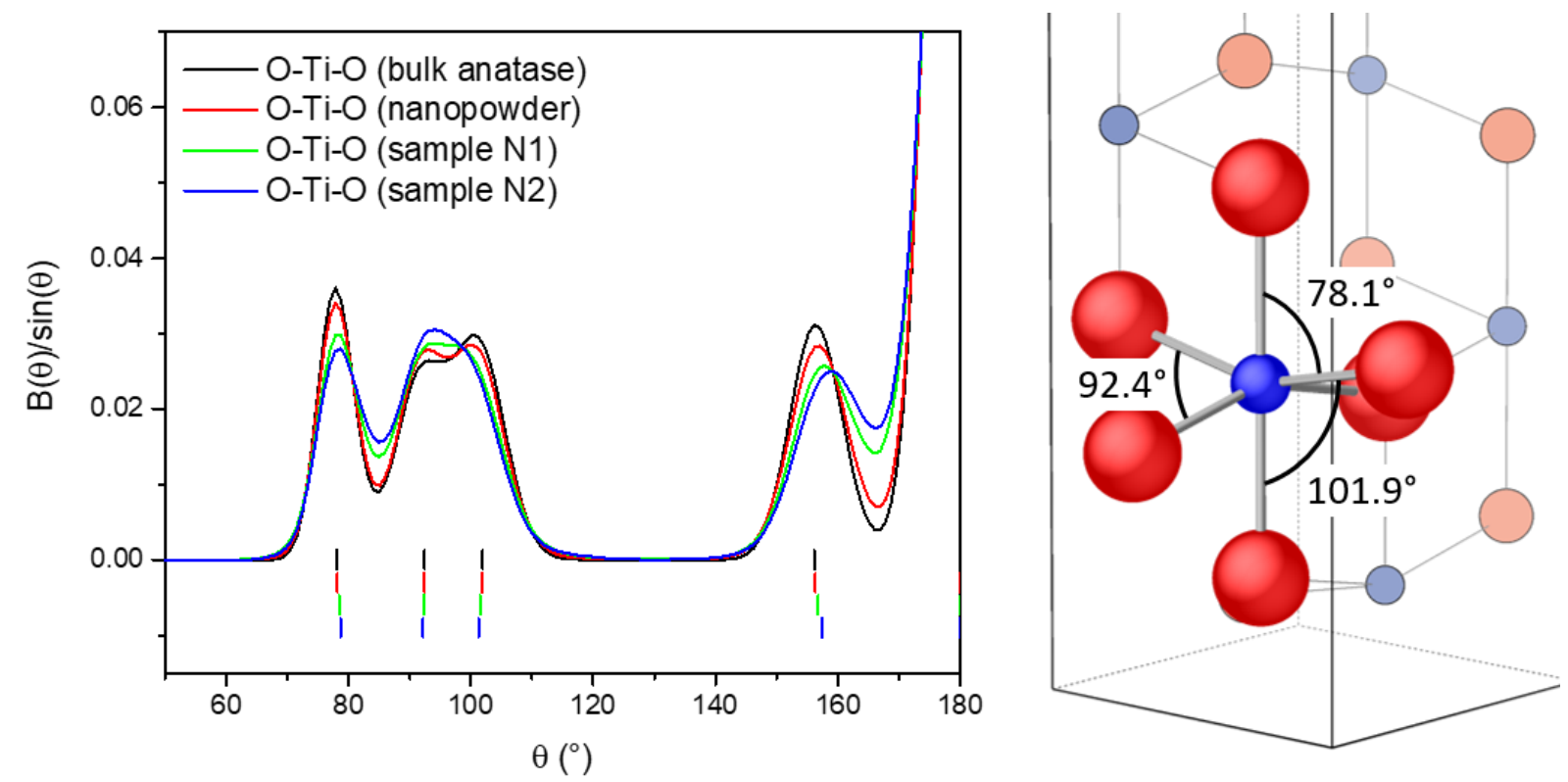

Figure 9: Left: O-Ti-O bond angle distributions for each of the 4 anatase samples, calculated from an ensemble of $100 \mathrm{RMC}$ refined configurations. Vertical tickmarks represent the average bond angles from the corresponding Rietveld refinements. Right: representations of the Ti-O octahedron in bulk anatase, with the three smaller O-Ti-O angles labelled.

To provide a visual representation of the relationship between the refined configurations and the crystal structure, each of the RMC configurations was collapsed down to the original unit cell dimensions. A comparison of each of the four samples is shown in Figure 10, and further orientations can be seen in Figures S8-S11. 


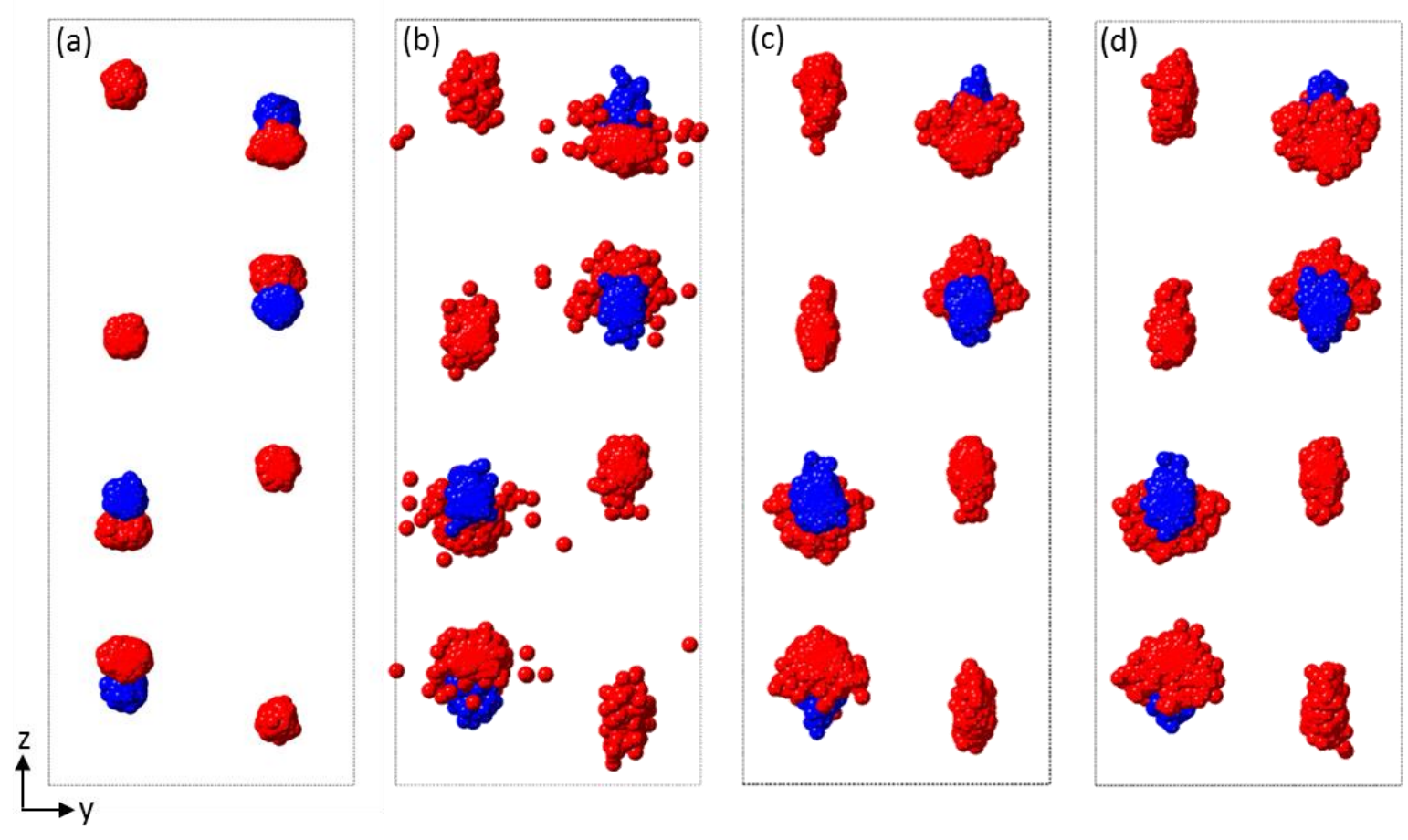

Figure 10: Collapsed RMC configurations for (a) bulk anatase, (b) commercial nanopowder, (c) sample N1 and (d) sample N2. Red spheres are $\mathrm{O}$ atoms, blue are $\mathrm{Ti}$ atoms.

The titanium atom "clouds" are small, with some elongation along the z-axis in the smaller nanoparticles. This is entirely in agreement with the anisotropic thermal displacement parameters (Figure 3). Conversely, the oxygen atom "clouds" for the nano-sized samples are larger and fan-shaped. Comparison with the largely spherical distributions in the bulk sample it is clear that a proportion of the oxygen atoms have shifted towards the plane of the titanium atoms.

Since the nano-sized $\mathrm{TiO}_{2}$ samples have a larger surface area to volume ratio than the bulk material, it is possible that the observed structural disorder is related to the surface region. Indeed a "core-shell" model is often proposed for $\mathrm{TiO}_{2}$ nanoparticles in which an ordered core of (usually) "anatase-like" structure is surrounded by a disordered shell $[1,26,40]$. Using the relationship defined by Chen et al. [16] the proportion of surface $\mathrm{TiO}_{2}$ is $12.5 / d$ (where $d$ is the diameter of the nanoparticle in Ångstroms), the exposed surface of the commercial nanopowder, samples $\mathrm{N} 1$ and $\mathrm{N} 2$ is $3.7 \%, 7.0 \%$ and $9.6 \%$ respectively. To link this with the RMC results, the magnitude of each oxygen atom's displacement from its ideal location was calculated, and the proportion that are displaced by more than $2.4 \AA$ corresponds to $1.41 \%$, $4.96 \%, 7.6 \%$ and $10.9 \%$ for each of our four samples. Because RMCProfile uses periodic boundary conditions, the model itself has no explicit surface regions, however the agreement between these two sets of numbers is compelling, and it seems likely that the observed disorder can be attributed to distortions on the surface of the particles.

It is interesting to consider the observed disorder in the context of the work of Coh et al. [41] who used density functional theory to identify a modification to the anatase structure which reduces the band gap. This modification can be understood by defining two types of [ $\left.\mathrm{TiO}_{6}\right]$ octahedra in the structure, which differ in the arrangement of the "equatorial" oxygen atoms (Figure S12). The pristine anatase structure is made up of alternating ( $A B A B A B$...) layers of these two octahedral geometries. Coh et al. [41] found that a structure with a repeated layer (i.e. ABAABA) was theoretically stable, and that it alters the valence band structure so as to reduce the overall band gap (Figure S12). A part of this structural representation is shown 
in Figure 11, along with the result if one of the oxygen atoms moves towards the Ti plane, as seen in the RMC results. Since each "equatorial" Ti-O bond is connected to another octahedron's "axial" bond, and the overall Ti-O bond length distribution changes very little, the structural modification can propagate as shown in Figure 11(b). While Coh et al. [41] discussed this modification in the context of "black" $\mathrm{TiO}_{2}$, which is produced at high temperatures in reducing conditions, the agreement between the theoretical result and the RMC results presented herein represents the first experimental evidence for this kind of structural modification in stoichiometric anatase nanoparticles.
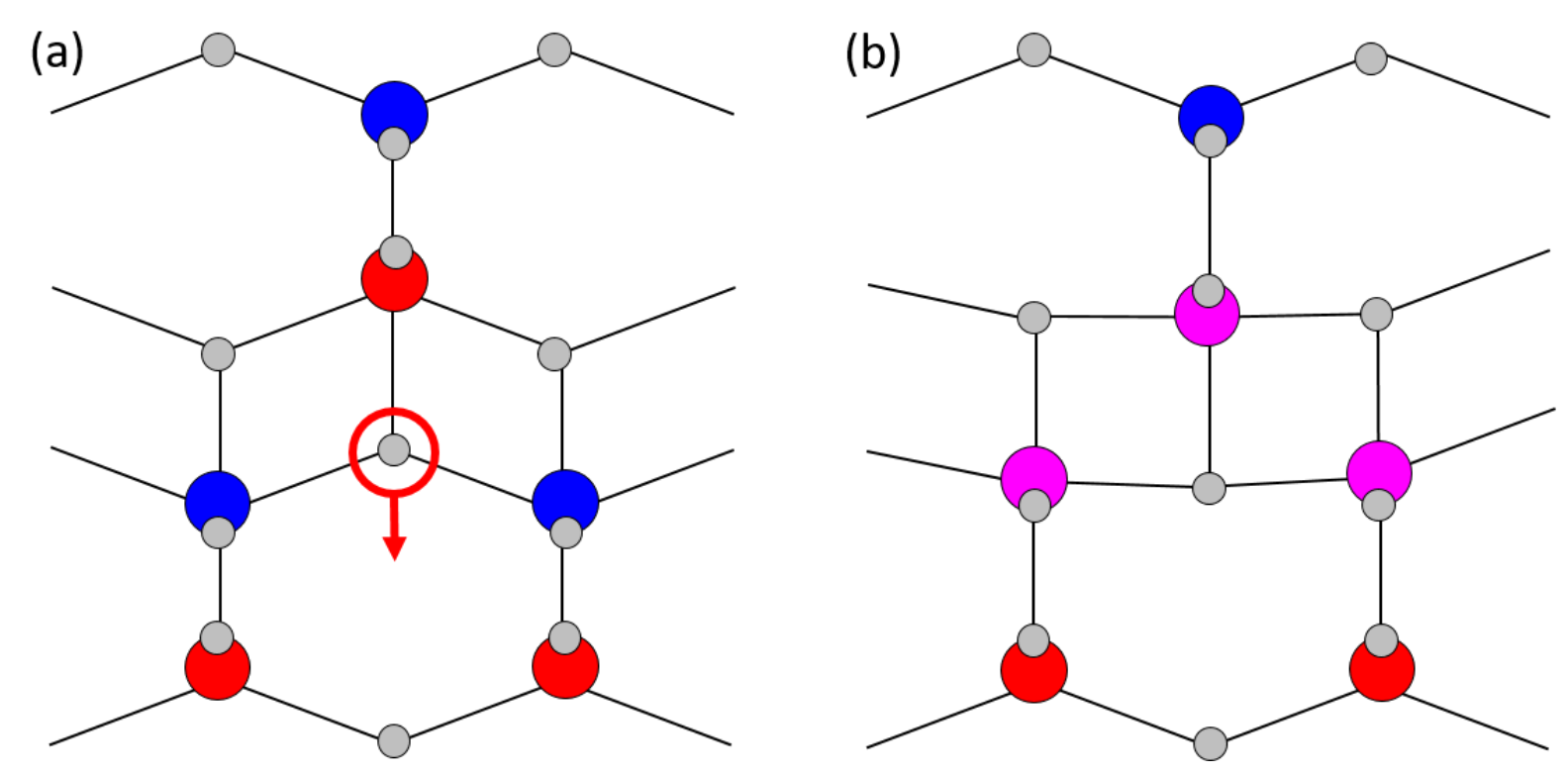

Figure 11: (a) A representation of the anatase structure showing the two types of titanium atoms as blue and red circles, respectively. Oxygen atoms are shown as small grey circles. The displacement of an oxygen atom in the z-direction is highlighted. (b) The result of the oxygen atom displacement which disrupts the alternating "red" and "blue" layers.

\section{Conclusions}

The structures of four samples of anatase $\mathrm{TiO}_{2}$ have been characterised using neutron diffraction, pair distribution function analysis and reverse Monte Carlo refinement. Structural distortion in the smaller nanoparticles is consistent with the displacement of a fraction of oxygen atoms roughly parallel to the z-axis and towards the plane formed by the titanium atoms. The number of displaced oxygen atoms increases with reduction in particle size and is consistent with the increased proportion of "exposed" surface in the smaller nanoparticles. The nature of the structural distortion results in a structure similar to that suggested by Coh et al. [41] as a modified form of anatase with a reduced band gap. This observation may prove important when attempting to understand the increased catalytic activity of smaller nanoparticles, and indeed shows that neutron total scattering is a tool capable of revealing subtle structural features that may have relevance in many functional materials.

\section{Acknowledgements}

I am grateful to STFC for the provision of beamtime on Polaris. Thanks are due to Matt Tucker and Ron Smith for their assistance with data collection, and to Steve Hull for helpful discussions. Computing resources were provided by STFC Scientific Computing Department's SCARF cluster.

\section{$\underline{\text { References }}$}

[1] X. Chen, S.S. Mao, Titanium Dioxide Nanomaterials: Synthesis, Properties, Modifications, and Applications, Chem. Rev. 107 (2007) 2891-2959. https://doi.org/10.1021/cr0500535. 
[2] A.F. Wells, Structural inorganic chemistry, 4th ed., Oxford University Press, London, 1975. https://doi.org/10.1038/157386a0.

[3] D. Reyes-Coronado, G. Rodríguez-Gattorno, M.E. Espinosa-Pesqueira, C. Cab, R. de Coss, G. Oskam, Phasepure $\mathrm{TiO}_{2}$ nanoparticles: anatase, brookite and rutile., Nanotechnology. 19 (2008) 145605. https://doi.org/10.1088/0957-4484/19/14/145605.

[4] T. a Kandiel, L. Robben, A. Alkaim, D. Bahnemann, Brookite versus anatase $\mathrm{TiO}_{2}$ photocatalysts: phase transformations and photocatalytic activities., Photochem. Photobiol. Sci. 12 (2013) 602-9. https://doi.org/10.1039/c2pp25217a.

[5] H. Zhang, J.F. Banfield, Understanding Polymorphic Phase Transformation Behavior during Growth of Nanocrystalline Aggregates: Insights from $\mathrm{TiO}_{2}$, J. Phys. Chem. B. 104 (2000) 3481-3487. https://doi.org/10.1021/jp000499j.

[6] A. Di Paola, M. Bellardita, L. Palmisano, Brookite, the Least Known $\mathrm{TiO}_{2}$ Photocatalyst, 2013. https://doi.org/10.3390/catal3010036.

[7] V. Chaudhary, A.K. Srivastava, J. Kumar, On the Sol-gel Synthesis and Characterization of Titanium Oxide Nanoparticles, MRS Proc. 1352 (2011) mrss11-1352-gg10-24. https://doi.org/10.1557/opl.2011.759.

[8] E. Silva Junior, F. a La Porta, M.S. Liu, J. Andrés, J. a Varela, E. Longo, A relationship between structural and electronic order-disorder effects and optical properties in crystalline $\mathrm{TiO}_{2}$ nanomaterials., Dalt. Trans. 44 (2015) 3159-75. https://doi.org/10.1039/c4dt03254c.

[9] S. Kelly, F.H. Pollak, M. Tomkiewicz, Raman Spectroscopy as a Morphological Probe for $\mathrm{TiO}_{2}$ Aerogels, J. Phys. Chem. B. 101 (1997) 2730-2734. https://doi.org/10.1021/jp962747a.

[10] D. Bersani, G. Antonioli, P.P. Lottici, T. Lopez, Raman study of nanosized titania prepared by sol-gel route, J. Non. Cryst. Solids. 232-234 (1998) 175-181. https://doi.org/10.1016/S0022-3093(98)00489-X.

[11] D. Bersani, P.P. Lottici, X. Ding, Phonon confinement effects in the Raman scattering by TiO 2 nanocrystals Phonon confinement effects in the Raman scattering by TiO 2 nanocrystals, Appl. Phys. Lett. 72 (1998) 73-75. https://doi.org/10.1063/1.120648.

[12] W.F. Zhang, Y.L. He, M.S. Zhang, Z. Yin, Q. Chen, Raman scattering study on anatase $\mathrm{TiO}_{2}$ nanocrystals, J. Phys. D. Appl. Phys. 33 (2000) 912-916. https://doi.org/10.1088/0022-3727/33/8/305.

[13] V. Luca, S. Djajanti, R.F. Howe, Structural and Electronic Properties of Sol-Gel Titanium Oxides Studied by Xray Absorption Spectroscopy, J. Phys. Chem. B. 102 (1998) 10650-10657. https://doi.org/10.1021/jp981644k.

[14] Z.Y. Wu, J. Zhang, K. Ibrahim, D.C. Xian, G. Li, Y. Tao, T.D. Hu, S. Bellucci, A. Marcelli, Q.H. Zhang, L. Gao, Z.Z. Chen, Structural determination of titanium-oxide nanoparticles by x-ray absorption spectroscopy, Appl. Phys. Lett. 80 (2002) 2973-2975. https://doi.org/10.1063/1.1470699.

[15] M. Wu, G. Lin, D. Chen, G. Wang, D. He, S. Feng, R. Xu, Sol-Hydrothermal Synthesis and Hydrothermally Structural Evolution of Nanocrystal Titanium Dioxide, Chem. Mater. 14 (2002) 1974-1980.

[16] L.X. Chen, T. Rajh, Z. Wang, M.C. Thurnauer, XAFS Studies of Surface Structures of $\mathrm{TiO}_{2}$ Nanoparticles and Photocatalytic Reduction of Metal Ions, J. Phys. Chem. B. 101 (1997) 10688-10697. https://doi.org/10.1021/jp971930g.

[17] V. Luca, Comparison of Size-Dependent Structural and Electronic Properties of Anatase and Rutile Nanoparticles, J. Phys. Chem. C. 113 (2009) 6367-6380. https://doi.org/10.1021/jp808358v.

[18] A.C. Hannon, W.S. Howells, A.K. Soper, ATLAS - A Suite of Programs for the Analysis of Time-of-flight Neutron Diffraction Data from Liquid and Amorphous Samples, Inst. Phys. Conf. Ser. 107 (1990) 193-211.

[19] D. Keen, A comparison of various commonly used correlation functions for describing total scattering, J. Appl. 
Crystallogr. 34 (2001) 172-177. https://doi.org/doi:10.1107/S0021889800019993.

[20] A.K. Soper, GudrunN and GudrunX : programs for correcting raw neutron and X-ray diffraction data to differential scattering cross section, Rutherford Applet. Lab. Tech. Rep. RAL-TR-201 (2011).

[21] M.G. Tucker, D.A. Keen, M.T. Dove, A.L. Goodwin, Q. Hui, RMCProfile: reverse Monte Carlo for polycrystalline materials, J. Phys. Condens. Matter. 19 (2007) 335218.

[22] C.A. Young, A.L. Goodwin, Applications of pair distribution function methods to contemporary problems in materials chemistry, J. Mater. Chem. 21 (2011) 6464. https://doi.org/10.1039/c0jm04415f.

[23] H.Y. Playford, L.R. Owen, I. Levin, M.G. Tucker, New Insights into Complex Materials Using Reverse Monte Carlo Modeling, Annu. Rev. Mater. Res. 44 (2014) 429-449. https://doi.org/10.1146/annurev-matsci-071312121712.

[24] S.J.L. Billinge, I. Levin, The problem with determining atomic structure at the nanoscale, Science (80-. ). 316 (2007) 561-565. https://doi.org/10.1126/science.1135080.

[25] V. Petkov, G. Holzhüter, U. Tröge, T. Gerber, B. Himmel, Atomic-scale structure of amorphous $\mathrm{TiO}_{2}$ by electron, X-ray diffraction and reverse Monte Carlo simulations, J. Non. Cryst. Solids. 231 (1998) 17-30. https://doi.org/http://dx.doi.org/10.1016/S0022-3093(98)00418-9.

[26] H. Zhang, B. Chen, J.F. Banfield, G.A. Waychunas, Atomic structure of nanometer-sized amorphous TiO ${ }_{2}$, Phys. Rev. B. 78 (2008) 214106.

[27] J.-L. Mi, K.M.Ø. Jensen, C. Tyrsted, M. Bremholm, B.B. Iversen, In situ total X-ray scattering study of the formation mechanism and structural defects in anatase $\mathrm{TiO}_{2}$ nanoparticles under hydrothermal conditions, CrystEngComm. 17 (2015) 6868-6877. https://doi.org/10.1039/C5CE00544B.

[28] M. Fernández-García, C. Belver, J.C. Hanson, X. Wang, J.A. Rodriguez, Anatase- $\mathrm{TiO}_{2}$ nanomaterials: Analysis of key parameters controlling crystallization, J. Am. Chem. Soc. 129 (2007) 13604-13612. https://doi.org/10.1021/ja074064m.

[29] G. Cernuto, N. Masciocchi, A. Cervellino, G.M.G.M. Colonna, A. Guagliardi, Size and Shape Dependence of the Photocatalytic Activity of $\mathrm{TiO}_{2}$ Nanocrystals: A Total Scattering Debye Function Study, J. Am. Chem. Soc. 133 (2011) 3114-3119. https://doi.org/10.1021/ja110225n.

[30] J. Liu, D. Olds, R. Peng, L. Yu, G.S. Foo, S. Qian, J. Keum, B.S. Guiton, Z. Wu, K. Page, Quantitative Analysis of the Morphology of $\{101\}$ and $\{001\}$ Faceted Anatase $\mathrm{TiO}_{2}$ Nanocrystals and Its Implication on Photocatalytic Activity, Chem. Mater. 29 (2017) 5591-5604. https://doi.org/10.1021/acs.chemmater.7b01172.

[31] X. Hua, Z. Liu, P.G. Bruce, C.P. Grey, The Morphology of $\mathrm{TiO}_{2}$ (B) Nanoparticles, J. Am. Chem. Soc. 137 (2015) 13612-13623. https://doi.org/10.1021/jacs.5b08434.

[32] T.A. Kandiel, A. Feldhoff, L. Robben, R. Dillert, D.W. Bahnemann, Tailored Titanium Dioxide Nanomaterials: Anatase Nanoparticles and Brookite Nanorods as Highly Active Photocatalysts, Chem. Mater. 22 (2010) 20502060. https://doi.org/10.1021/cm903472p.

[33] D. Shi, Z. Li, Y. Zhang, X. Kou, L. Wang, J. Wang, J. Li, Synthesis and characterizations of amorphous titania, Nanosci. Nanotechnol. Lett. 1 (2009) 165-170.

[34] O. Arnold, J.C. Bilheux, J.M. Borreguero, A. Buts, S.I. Campbell, L. Chapon, M. Doucet, N. Draper, R. Ferraz Leal, M.A. Gigg, V.E. Lynch, A. Markvardsen, D.J. Mikkelson, R.L. Mikkelson, R. Miller, K. Palmen, P. Parker, G. Passos, T.G. Perring, P.F. Peterson, S. Ren, M.A. Reuter, A.T. Savici, J.W. Taylor, R.J. Taylor, R. Tolchenov, W. Zhou, J. Zikovsky, Mantid - Data analysis and visualization package for neutron scattering and $\mu S R$ experiments, Nucl. Instruments Methods Phys. Res. Sect. A Accel. Spectrometers, Detect. Assoc. Equip. 764 (2014) 156-166. https://doi.org/10.1016/j.nima.2014.07.029.

[35] A.C. Larson, R.B. Von Dreele, General Structure Analysis System (GSAS), Los Alamos Natl. Lab. Rep. LAUR 86- 
748. (1994).

[36] B.H. Toby, EXPGUI, a graphical user interface for GSAS, J. Appl. Crystallogr. 34 (2001) 210-213.

[37] C.L. Farrow, P. Juhas, J.W. Liu, D. Bryndin, E.S. Bozin, J. Bloch, T. Proffen, S.J. Billinge, PDFfit2 and PDFgui: computer programs for studying nanostructure in crystals, J Phys Condens Matter. 19 (2007) 335219. https://doi.org/10.1088/0953-8984/19/33/335219.

[38] I.E. Grey, N.C. Wilson, Titanium vacancy defects in sol-gel prepared anatase, J. Solid State Chem. 180 (2007) 670-678. https://doi.org/10.1016/j.jssc.2006.11.028.

[39] H. Zhu, Q. Li, Y. Ren, L. Fan, J. Chen, J. Deng, X. Xing, Hydration and Thermal Expansion in Anatase Nanoparticles, Adv. Mater. 28 (2016) 6894-6899. https://doi.org/10.1002/adma.201600973.

[40] S. Sorcar, Y. Hwang, C.A. Grimes, S. II In, Highly enhanced and stable activity of defect-induced titania nanoparticles for solar light-driven $\mathrm{CO} 2$ reduction into CH4, Mater. Today. 20 (2017) 507-515. https://doi.org/10.1016/j.mattod.2017.09.005.

[41] S. Coh, P.Y. Yu, Y. Aoki, S. Saito, S.G. Louie, M.L. Cohen, Alternative structure of $\mathrm{TiO}_{2}$ with higher energy valence band edge, Phys. Rev. B. 95 (2017) 1-7. https://doi.org/10.1103/PhysRevB.95.085422. 